ARTICLE

https://doi.org/10.1038/s41467-020-14456-0

\title{
Structural basis for electron transport mechanism of complex I-like photosynthetic NAD(P)H dehydrogenase
}

\author{
Xiaowei Pan (10) ${ }^{1,5}$, Duanfang Cao (iD ${ }^{1,5}$, Fen Xie ${ }^{1,2,5}$, Fang $\mathrm{Xu}^{2,3,5}$, Xiaodong Su${ }^{1}$, Hualing $\mathrm{Mi}^{3 *}$, \\ Xinzheng Zhang (1D) ${ }^{1,2,4 \star} \&$ Mei Li (i) ${ }^{1 \star}$
}

$\mathrm{NAD}(\mathrm{P}) \mathrm{H}$ dehydrogenase-like (NDH) complex NDH-1L of cyanobacteria plays a crucial role in cyclic electron flow (CEF) around photosystem I and respiration processes. NDH-1L couples the electron transport from ferredoxin $(\mathrm{Fd})$ to plastoquinone $(\mathrm{PQ})$ and proton pumping from cytoplasm to the lumen that drives the ATP production. NDH-1L-dependent CEF increases the ATP/NADPH ratio, and is therefore pivotal for oxygenic phototrophs to function under stress. Here we report two structures of NDH-1L from Thermosynechococcus elongatus $\mathrm{BP}-1$, in complex with one $\mathrm{Fd}$ and an endogenous $\mathrm{PQ}$, respectively. Our structures represent the complete model of cyanobacterial $\mathrm{NDH}-1 \mathrm{~L}$, revealing the binding manner of $\mathrm{NDH}-1 \mathrm{~L}$ with $\mathrm{Fd}$ and $\mathrm{PQ}$, as well as the structural elements crucial for proper functioning of the NDH-1L complex. Together, our data provides deep insights into the electron transport from $\mathrm{Fd}$ to $\mathrm{PQ}$, and its coupling with proton translocation in $\mathrm{NDH}-1 \mathrm{~L}$.

\footnotetext{
${ }^{1}$ National Laboratory of Biomacromolecules, CAS Center for Excellence in Biomacromolecules, Institute of Biophysics, Chinese Academy of Sciences, Beijing 100101, PR China. ${ }^{2}$ University of Chinese Academy of Sciences, Beijing 100049, PR China. ${ }^{3}$ National Key Laboratory of Plant Molecular Genetics, Institute of Plant Physiology and Ecology, Shanghai Institutes for Biological Sciences, Chinese Academy of Science, Shanghai 200032, PR China. ${ }^{4}$ Center for Biological Imaging, CAS Center for Excellence in Biomacromolecules, Institute of Biophysics, Chinese Academy of Sciences, Beijing 100101, PR China. ${ }^{5}$ These authors contributed equally: Xiaowei Pan, Duanfang Cao, Fen Xie, Fang Xu. *email: hlmi@sibs.ac.cn; xzzhang@ibp.ac.cn; meili@ibp.ac.cn
} 
$\mathrm{D}$ uring oxygenic photosynthesis, light energy is utilized by photosystems I and II (PSI and PSII) to drive the electron transport, which can be classified into two types, the linear electron transport/flow and the cyclic electron transport/ flow $^{1,2}$. During LEF, electrons are extracted from water molecules by PSII and transported to PSI through cytochrome $b_{6} f$ (Cyt $\left.b_{f} f\right)$ and free electron carrier including plastoquinone $(\mathrm{PQ})$, before being ultimately used to reduce $\mathrm{NADP}^{+}$to NADPH. Simultaneously, a proton gradient across the thylakoid membrane is generated and drives the ATP synthase to produce ATP ${ }^{3}$. Both $\mathrm{NADPH}$ and ATP are required for the subsequent $\mathrm{CO}_{2}$ assimilation during the Calvin-Bensen-Bassham cycle and other cellular metabolisms $\mathrm{s}^{2,3}$. During the CEF process, the electrons are recycled between PSI and Cyt $b_{6} f$ through PQ pool. CEF functions without PSII, simply creating the transmembrane proton gradient, which allows only ATP production without generating $\mathrm{NADPH}^{4,5}$. LEF alone probably generates insufficient ATP to balance the ATP/NADPH consumption for $\mathrm{CO}_{2}$ assimilation $^{6-8}$, whereas $\mathrm{CEF}$ increases the produced ATP/NADPH ratio ${ }^{9}$, thus is important for achieving high efficiency of the Calvin-Bensen-Bassham cycle, especially under multiple stressed conditions $s^{4,10-12}$, as well as for C4 photosynthesis ${ }^{13,14}$. In addition, photosynthetic organisms that are exposed to environmental stress usually exhibit increased demand for ATP ${ }^{15}$, therefore, CEF is crucial for the quick response of phototrophs to the changing environment. Furthermore, CEF plays an essential role in photoprotection by generating a large transmembrane proton gradient, thereby inducing energy dependent quenching to dissipate excessively absorbed energy ${ }^{9,16-19}$.

In both cyanobacteria and plants, a type-I $\mathrm{NAD}(\mathrm{P}) \mathrm{H}$ dehydrogenase-like (NDH-1) complex is crucial for CEF and respiration. NDH-1 complex accepts electrons from electron carrier ferredoxin $(\mathrm{Fd})$ or $\mathrm{NAD}(\mathrm{P}) \mathrm{H}$ in PSI acceptor side, and transfers them to Cyt $b_{6} f$ through $\mathrm{PQ}^{20,21}$. NDH-dependent CEF was previously suggested to fine-tune the chloroplast redox state, and to prevent overreduction of the stroma under stress ${ }^{2,14}$. The NDH-1 complexes belong to the NAD(P)H:Quinone oxidoreductase family that includes the complex I (NADH dehydrogenase) of the respiratory chain in bacteria and mitochondria ${ }^{15,22}$. Cyanobacteria possess several types of NDH-1 complexes, namely NDH-1L/1L' (large), NDH-1M (medium), and $\mathrm{NDH}-1 \mathrm{~S} / 1 \mathrm{~S}^{\prime}$ (small), differing in size and the isoforms of $\mathrm{NdhD} / \mathrm{NdhF}$ subunits, and the latter two types can further associate into NDH-1MS/NDH-1MS'23,24. NDH-1L containing NdhD1/NdhF1 is involved in CEF around PSI and respiration ${ }^{15,20}$. The NDH-1L complex and bacterial complex I form a similar L-shaped architecture and share 11 conserved subunits. In addition, cyanobacterial NDH-1L possesses eight oxygenic photosynthesis-specific (OPS) subunits ${ }^{25}$, namely $\mathrm{NdhL}^{26}, \mathrm{NdhM}, \mathrm{NdhN}^{27}, \mathrm{NdhO}^{28}, \mathrm{NdhP}, \mathrm{NdhQ}^{29}, \mathrm{NdhS}^{30}$, and $\mathrm{NdhV}^{31,32}$, but lacks three hydrophilic subunits, which contain the NADH binding site in bacterial complex $\mathrm{I}^{33}$. These earlier results were confirmed by recent studies showing that the photosynthetic NDH complex binds Fd as the electron donor, rather than NADPH ${ }^{34,35}$. The electron delivered from Fd is eventually transferred to $\mathrm{PQ}$ which is bound within the NDH complex.

Two structures of NDH-1L from the thermophilic cyanobacterium Thermosynechococcus elongatus BP-1 were recently reported ${ }^{35,36}$. These studies identified the structures and locations of 18 subunits (lacking $\mathrm{NdhV}$ ), providing solid foundations for better understanding the architecture and assembly of the $\mathrm{NDH}-1 \mathrm{~L}$ complex. NDH-1L in these two structures adopts a characteristic L-shaped architecture, which can be divided into membrane arm/domain and hydrophilic arm/domain, composed of ten and eight subunits, respectively. However, the structure and accurate location of the soluble subunit NdhV, which might be loosely associated with the complex ${ }^{31,32}$, remain unknown. Moreover, it was previously suggested that NdhS participates in $\mathrm{Fd}$ binding 34,37 , while another recent study proposed an alternative $\mathrm{Fd}$ binding pocket formed by $\mathrm{NdhO}^{36}$. To date, the exact $\mathrm{Fd}$ binding site in cyanobacterial NDH-1L remains to be elucidated. In addition, as only the apo form of NDH-1L was structurally characterized, the detailed interactions of $\mathrm{PQ}$ within photosynthetic NDH complex has not been determined, although a quinone-reaction chamber ( $Q$ chamber) was previously suggested in respiratory complex I, based on the substrate/inhibitor-bound structures from Thermus thermophilus (Tt complex I) ${ }^{38}$ and Yarrowia lipolytica ( $\mathrm{Yl}$ complex I) ${ }^{39}$. To further advance our understanding of NDH functionality, we solved two single particle cryo-electron microscopy (cryo-EM) structures of NDH-1L from T. elongatus BP-1. Our results provide the structural model of $\mathrm{NDH}-1 \mathrm{~L}$ containing NdhV, and reveal the detailed binding modes of Fd and PQ. Moreover, we observe a variety of conformational changes between our structure and previously reported $\mathrm{NDH} /$ complex I structures, thus shedding light on the electron transport process and its coupling with proton translocation.

\section{Results}

Overall structure. We first solved the structure of NDH-1L complex purified from T. elongatus BP-1 (Supplementary Fig. 1) at an overall resolution of $3 \AA$ (Supplementary Fig. 2, Table 1). Interestingly, we found that the NDH-1L complex binds a PQ

Table 1 Cryo-EM data collection and refinement and validation statistics.

\section{NDH-PQ (EMDB- NDH-Fd (EMDB-} 9990, PDB 6KHJ) 9989, PDB 6KHI)

Data collection and processing

Magnification

Voltage (kV)

Electron exposure $\left(e^{-} / \AA^{2}\right)$

Defocus range $(\mu \mathrm{m})$

Pixel size $(\AA)$

Symmetry imposed

Initial particle images (no.)

130,000

300

60

$0.5-2.5$

1.04

C1

Final particle images (no.)

434,021

130,000

Map resolution $(\AA)$

FSC threshold

187,788

3.0

Map resolution range $(\AA)$

0.143

Refinement

Initial model used (PDB code) 4HEA

Model resolution $(\AA)$

FSC threshold

3.0

0.5

Map sharpening $B$ factor $\left(\AA^{2}\right) \quad-98$

Model composition

Nonhydrogen atoms $\quad 31,027$

31,027
3877

Protein residues

20

300

Ligands

B factors $\left(\AA^{2}\right)$

Protein

Ligand

45.0

48.5

60

$0.5-2.5$

1.04

C1

276,859

152,003

3.2

0.143

2.8-4.4

$\mathrm{NDH}-\mathrm{PQ}, 5 \mathrm{AUI}$

3.2

0.5

$-106$

R.m.s. deviations

Bond lengths $(\AA)$

0.007

32,635

4095

19

Bond angles $\left({ }^{\circ}\right)$

0.999

55.6

60.4

Validation

MolProbity score

Clash score

1.86

Poor rotamers (\%)

6.84

0.41

0.006

0.945

Ramachandran plot

Favored (\%)

Allowed (\%)

92.13

Disallowed (\%)

7.85

0.02
1.92

7.31

0.27

91.04

8.94

0.02 

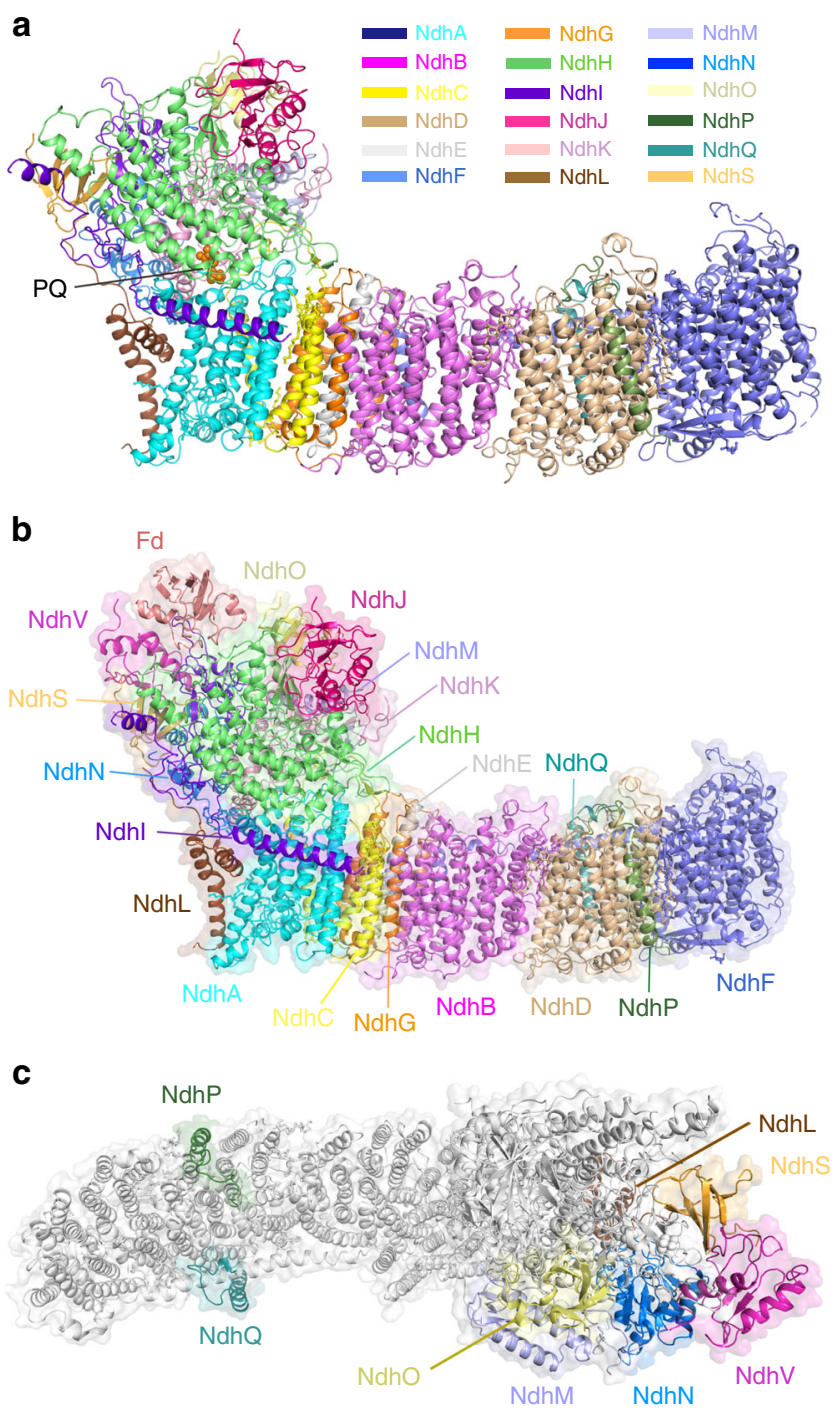

Fig. 1 The NDH-PQ and NDH-Fd structures. a Cartoon representation of the NDH-PQ structure viewed along the membrane plane. PQ is shown as orange spheres and indicated. b The overall structure of NDH-Fd viewed along the membrane plane. c The location of OPS subunits in NDH-1L complex. The eight OPS subunits are colored differently and indicated, while other subunits are shown in white.

molecule at a pocket above the membrane plane (Fig. 1a). We assume that this is an endogenous PQ molecule, which was trapped in the cyanobacterial NDH-1L complex during purification, as was earlier reported for Escherichia coli complex I ${ }^{40}$. Thus, we named this structure as NDH-PQ. Similar to the two previously reported cyanobacterial NDH-1L structures ${ }^{35,36}$, our NDH-PQ structure also contains 18 subunits (Fig. 1a), without any signs of association with $\mathrm{NdhV}$. These results indicated that $\mathrm{NdhV}$ is loosely associated with other NDH subunits, in line with previous biochemical data ${ }^{31,32}$. In addition, we found that the NdhS subunit in the NDH-PQ structure exhibits weak cryo-EM density, suggesting that NdhS is also loosely associated. Previous reports showed that $\mathrm{NdhV}$ interacts with $\mathrm{NdhS}$ and requires NdhS for its stabilization 31,32 . Therefore, to obtain the intact $\mathrm{NDH}-1 \mathrm{~L}$ in complex with $\mathrm{Fd}$, we expressed and purified the proteins of $\mathrm{Fd}, \mathrm{NdhV}$, and $\mathrm{NdhS}$ from T. elongatus BP-1 via E. coli (Supplementary Fig. 1) and incubated the purified proteins with NDH-1L in order to increase their occupancy, and finally solved the structure of $\mathrm{NDH}-1 \mathrm{~L}$ containing $\mathrm{NdhV}$ and in complex with Fd (NDH-Fd) at $3.2 \AA$ resolution (Supplementary Fig. 3, Table 1). The NDH-Fd structure, highly similar with the NDH-PQ structure, contains all $19 \mathrm{NDH}$ subunits, and binds one Fd molecule at the top of the hydrophilic arm (Fig. 1b). Supplementary Fig. 4 shows the representative cryo-EM densities of Fd and several subunits and cofactors of NDH-1L complex.

The membrane arm with lipid and carotenoid molecules. The membrane arm in NDH-PQ and NDH-Fd structures are almost identical, seven subunits (NdhA-G) conserved in respiratory complex I constitute the membrane core, and three OPS subunits (NdhL, NdhP-Q) attach to the peripheral region. NdhP and NdhQ are located at the distal part, sandwiching NdhD from opposite sides, whereas $\mathrm{NdhL}$ is positioned adjacent to NdhA (Fig. 1c). Furthermore, we modeled two carotenoids and thirteen lipid molecules (Supplementary Fig. 4g-1) within the membrane arm in both NDH-PQ and NDH-Fd structures (Fig. 2). In addition to the previously described protein-protein interaction 35,36 , these nonprotein components greatly contribute to the integrity of the membrane arm. At the proximal region, two digalactosyldiacyl glycerol (DGDG) molecules fill the empty space between NdhA and NdhL from the luminal side, with one DGDG further hydrogen bonded with the N-terminal region of NdhC. In the vicinity of the two DGDGs, another lipid molecule is sandwiched by the first transmembrane helix (TMH) of NdhC and NdhG. Therefore, the three lipid molecules function in linking the three proximal subunits at the lumen (Fig. 2b). At the distal region, seven lipid molecules were observed, and six are located at the stromal side (Fig. 2a). Among the seven lipids, four and two are found to gather at the NdhF-NdhD, $\mathrm{NdhD}-\mathrm{NdhB}$ interfaces, respectively (Fig. 2c). Moreover, two carotenoid (modeled as $\beta$-carotene) molecules greatly enhance the NdhF-NdhD interaction. One $\beta$-carotene (BCR1) is located near NdhP and sandwiched by two lipid molecules (Fig. 2d), together forming a cluster and serving as molecular glue, as was previously suggested by a NDH-1L structural study ${ }^{35}$. On the opposite side close to NdhQ, the second $\beta$-carotene (BCR2) molecule is almost parallel to the membrane plane and binds in a pocket below the horizontal helix of NdhF (Fig. 2e). This observed $\beta$-carotene secures the $\mathrm{NdhF}-\mathrm{NdhD}$ interaction, comparable to a bolt-like feature. In addition to their function for light harvesting and energy quenching, carotenoid molecules are also crucial for the inter-subunit interaction and stabilization for the photosynthetic complexes ${ }^{41}$. The two $\beta$-carotene molecules result in the strong association between $\mathrm{NdhF}$ and $\mathrm{NdhD}$, thus explaining the presence of a smaller NDH-1M complex in cyanobacteria, which lacks both $\mathrm{NdhD}$ and $\mathrm{NdhF}$ subunits ${ }^{42,43}$. In addition, our structures indicate that both NdhP and NdhQ form direct contacts with $\mathrm{NdhD}$ and $\mathrm{NdhF}$, and further interact with interfacial lipids and carotenoids (Fig. 2d), thus contribute to the stabilization of NDH-1L complex, in line with the finding from previous reports that deletion of each subunit disassembles the NDH-1L complex ${ }^{44,45}$.

The hydrophilic arm and the interface between two arms. The NDH-Fd structure contains the complete hydrophilic arm composed of nine subunits, including $\mathrm{NdhV}$ (Fig. 3a), which was absent in both previously reported NDH structures. The four subunits conserved in respiratory complex I ( $\mathrm{NdhH}-\mathrm{K})$ form the hydrophilic core of NDH-1L. Among them, NdhI and NdhK harbor two and one $4 \mathrm{Fe}-4 \mathrm{~S}$ clusters (corresponding to the clusters N6a, N6b, and N2 from the respiratory complex I) essential for electron transport (Figs. 2a, 3a). Five OPS subunits (NdhM, $\mathrm{NdhN}, \mathrm{NdhO}, \mathrm{NdhS}$, and $\mathrm{NdhV}$ ) are located at the peripheral region, surrounding half of the hydrophilic core (Fig. 1c). 
a
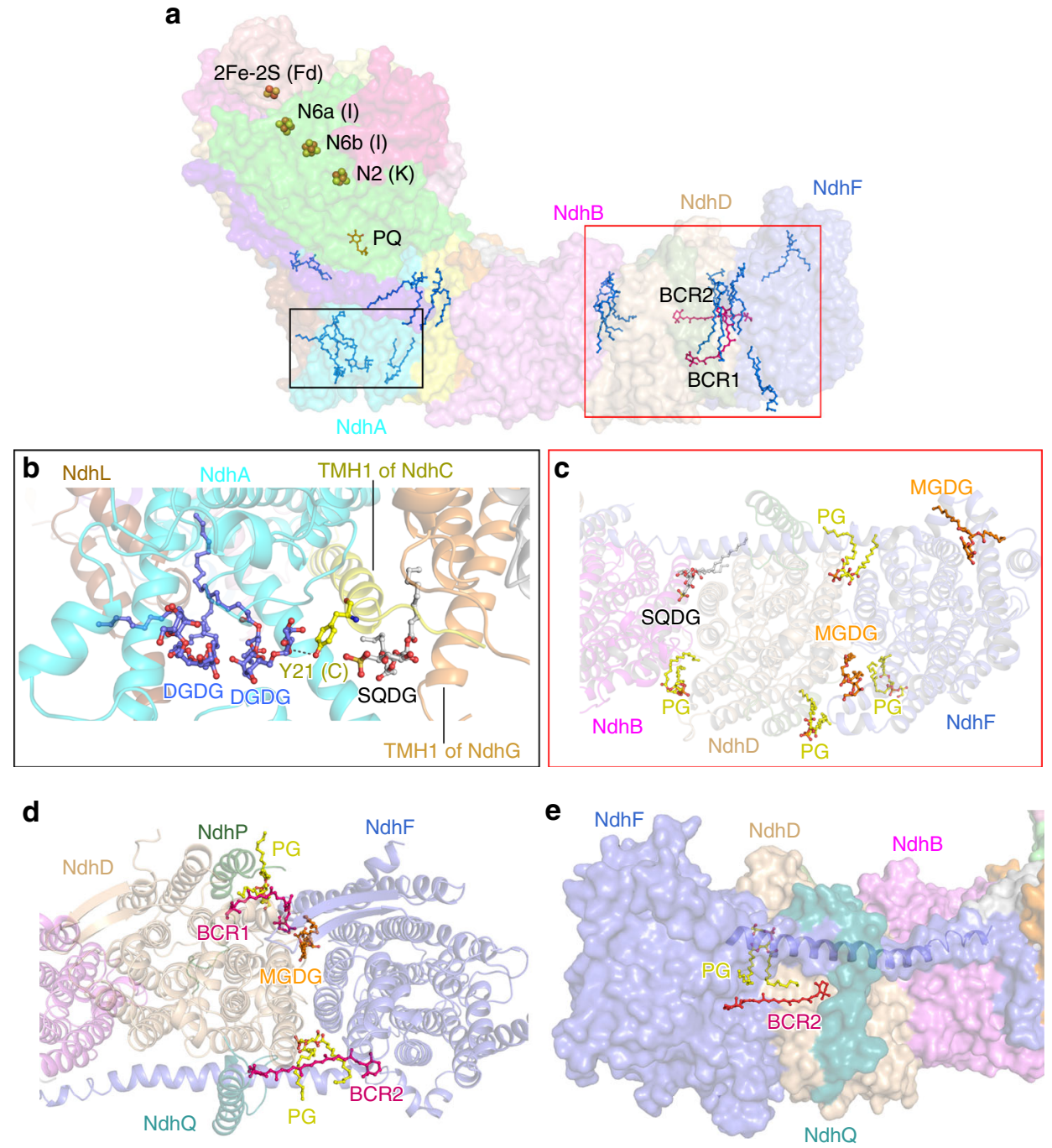

Fig. 2 The membrane arm with lipid and carotenoid molecules. a Distribution of cofactors in NDH-Fd structure. Fe-S clusters are shown as spheres, PQ (orange), lipids (blue), and carotenoids (red) are shown as sticks. b Three lipid molecules (shown as ball-and-stick mode) contribute to the stabilization of NdhL, NdhA, NdhC, and NdhG. c The stromal side view of the lipid distribution at the distal part of the membrane arm. d Two carotenoid (BCR) and three lipid molecules participate in NdhF-NdhD interactions. e The binding pocket of BCR2. The color codes of subunits are the same as those in Fig. 1. The horizontal helix of NdhF is shown as blue cartoon. The BCR molecules are shown as red sticks. Lipid molecules are colored yellow for phosphatidyl glycerol $(P G)$, gray for sulfoquinovosyldiacyl glycerol (SQDG), orange for monogalactosyldiacyl glycerol (MGDG), and blue for DGDG in b-e.

Extensive interactions are formed between the conserved subunits and OPS subunits.

$\mathrm{NdhV}$ is composed of central loops/ $\beta$-strands, which is flanked by the $\mathrm{N}$ - and C-terminal helix at one side (Fig. 3b, Supplementary Fig. 4a). In the NDH-Fd structure, NdhV is located above $\mathrm{NdhN}$ and NdhS (Fig. 3c). The central loops/ $\beta$-strands of NdhV join with the loops/ $\beta$-strands of NdhS (Supplementary Fig. 5a), forming strong interactions. This structural observation is in line with previous results suggesting that $\mathrm{NdhV}$ requires $\mathrm{NdhS}$ for its binding and stabilization ${ }^{31,32}$. In addition, NdhV, NdhS, and $\mathrm{NdhN}$ contact each other and the three OPS subunits together form a triangular structure, simultaneously interacting with NdhI (Supplementary Fig. 5b). Particularly, NdhV uses its C-terminal helix to associate with the long hairpin loop of $\mathrm{NdhI}$ (Supplementary Fig. 5c), which projects into the cytoplasmic region above the hydrophilic arm (Fig. 3d). The close association between NdhV and NdhI observed in the structure agrees with an earlier report that $\mathrm{NdhV}$ is crosslinked with $\mathrm{NdhI}^{31}$.

In NDH complex, the soluble subunits $\mathrm{NdhK}$ and $\mathrm{NdhH}$ together with the membrane subunit NdhA play major role in maintaining the association between the membrane and hydrophilic arms (Supplementary Fig. 6a). In addition, NdhI, NdhC, and the OPS subunit NdhL also contribute to the interaction between the two arms. The cytoplasmic loop between the first and second TMH (TMH1-2 loop) of NdhC (Supplementary Fig. 4d) contacts NdhA and the soluble subunits NdhK/NdhH/NdhM (Supplementary Fig. 6b), reinforcing the interactions between the two arms. Moreover, the membrane subunit NdhL has a Cterminal fragment projecting into the cytoplasmic region and associating with $\mathrm{NdhI} / \mathrm{NdhN}$ (Supplementary Fig. 6c). While the soluble NdhI possesses an N-terminal amphiphilic helix (N-helix) that embraces the membrane subunits NdhA/NdhL (Supplementary Fig. 6c). In addition, three lipid molecules were found at the interface between the two arms, facilitating their association (Supplementary Fig. 6d, e). Two of these lipids link the N-helix of NdhI with the membrane domain of $\mathrm{NdhA}$ and $\mathrm{NdhC}$ (Supplementary Fig. 6d), whereas the third lipid is located in an enclosed cavity formed by the $\mathrm{N}$-terminal fragments from NdhL, NdhN, and NdhK (Supplementary Fig. 6e). Our structure highlights the pivotal role of lipid molecules in 
a

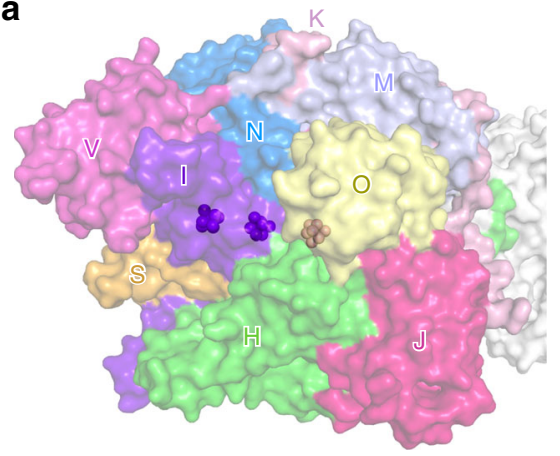

C

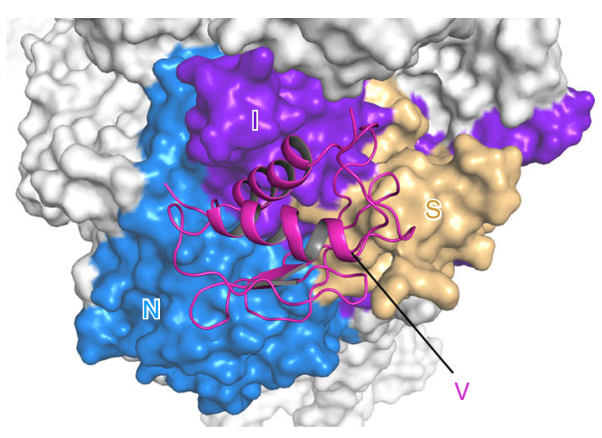

b

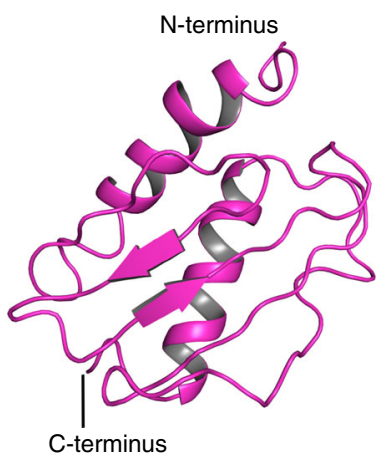

d

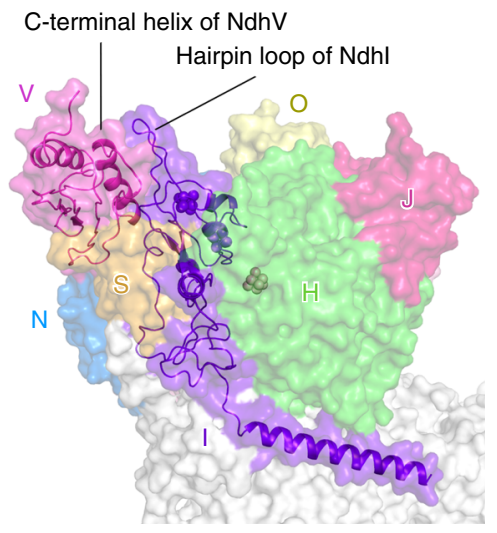

Fig. 3 The hydrophilic arm containing NdhV. a The location of subunits in the hydrophilic domain. The nine NDH subunits in the hydrophilic domain are colored differently and labeled with the abbreviations of one letter (for example, letter $\mathrm{V}$ indicates subunit NdhV). The $4 \mathrm{Fe}-4 \mathrm{~S}$ clusters are shown as spheres. b Overall structure of NdhV. c The binding position of NdhV. NdhV is shown in cartoon, other subunits are shown in surface mode. Ndhl, NdhS, and $\mathrm{NdhN}$ are colored differently and labeled, whereas other subunits are shown in white. $\mathbf{d}$ The C-terminal helix of NdhV interacts with the hairpin loop of Ndhl.

connecting the hydrophilic arm with the membrane arm of the $\mathrm{NDH}$ complex.

The Fd binding pocket. Several OPS subunits, including NdhS, $\mathrm{NdhO}$, and $\mathrm{NdhV}$, were previously suggested to be involved in $\mathrm{Fd}$ binding $32,34,36$. Surprisingly, neither of these subunits directly interact with Fd as shown in our NDH-Fd structure, whereas two conserved subunits, $\mathrm{NdhI}$ and $\mathrm{NdhH}$, form extensive contacts with Fd. The long hairpin loop of NdhI, together with a Cterminal fragment from $\mathrm{NdhH}$, shapes a positively-charged patch on the surface of NDH-1L complex, and forms strong electronic interactions with the acidic residues of Fd (Fig. $4 \mathrm{a}-\mathrm{c}$ ), thus is essential for Fd binding. This structural feature is consistent with previous studies showing that Fd features a highly acidic surface, with recruitment usually driven by basic patches on the binding partner $^{46-48}$. Furthermore, our surface plasmon resonance (SPR) results demonstrated that the binding affinity of NDH-1L with Fd is significantly increased at higher $\mathrm{pH}$ (Supplementary Fig. 7), probably because the stronger interaction between the acidic Fd and the basic NDH complex.

In addition to $\mathrm{NdhI}$ and $\mathrm{NdhH}$, several OPS subunits also contribute to the Fd binding, though they lack any direct contacts with Fd. In our NDH-Fd structure, NdhV and Fd are almost symmetrically distributed along opposite sides of the hairpin loop of NdhI (Fig. 4d), thus NdhV provides strong support for the hairpin loop. Two positively-charged residues, which are located on the hairpin loop and highly conserved in NdhI, are hydrogen bonded with residues from Fd. Superposition of our NDH-Fd and NDH-PQ structures revealed a swing of the hairpin loop toward $\mathrm{Fd}$ in NDH-Fd structure (Fig. 4e). This observation suggests
NdhV may enhance the NdhI-Fd interaction by simultaneously binding the long hairpin loop of NdhI, which provides a rationale for previous data suggesting that $\mathrm{NdhV}$ is pivotal for CEF activity $^{31,32}$. Moreover, by contacting $\mathrm{NdhI}$ and/or $\mathrm{NdhH}$ through their loop regions, NdhS and NdhN may also contribute to the formation of the Fd binding pocket (Fig. 4f). The interactions between $\mathrm{Fd}$ and $\mathrm{NDH}$ complex position the $2 \mathrm{Fe}-2 \mathrm{~S}$ cluster of Fd close to the N6a cluster of NdhI, with a distance of $9.6 \AA$ (Fig. 4c). This distance is comparable to those between other $4 \mathrm{Fe}-4 \mathrm{~S}$ clusters in NDH-1L complex, and could enable efficient electron transport ${ }^{49,50}$.

The PQ chamber. In the NDH-PQ structure, we modeled a PQ molecule in a pocket (PQ chamber) at the interface of the membrane and hydrophilic arms (Fig. 1a). It is noteworthy that the PQ molecule exhibits weaker density compared with other stable parts of the NDH structure, indicating that either the PQ molecule is mobile or a portion of NDH complex in our preparation does not bind PQ. The head group of PQ is oriented toward the N2 cluster (Fig. 5), and the end of its tail is invisible in the cryo-EM map (Supplementary Fig. 4f). The four-helix bundle region and the loop between the first two $\beta$-stands ( $\beta 1-2$ loop) of $\mathrm{NdhH}$, the fragment around the second helix of NdhK, and the loop connecting TMHs 5 and 6 (TMH5-6 loop) of NdhA are the major structural elements that constitute the PQ chamber (Fig. 5b). These fragments are further stabilized by subunits NdhI, $\mathrm{NdhL}$, and NdhC (Fig. 5b). Interestingly, these six subunits are also essential for maintaining the association between the membrane and hydrophilic arms (Supplementary Fig. 6a-c). Within the chamber, the head group of PQ molecule is located $\sim 18 \AA$ 
a

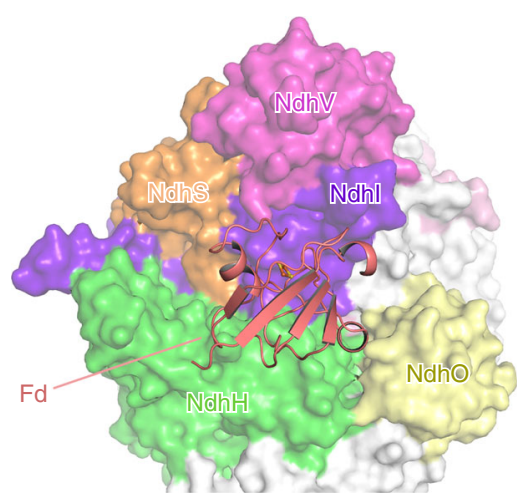

b

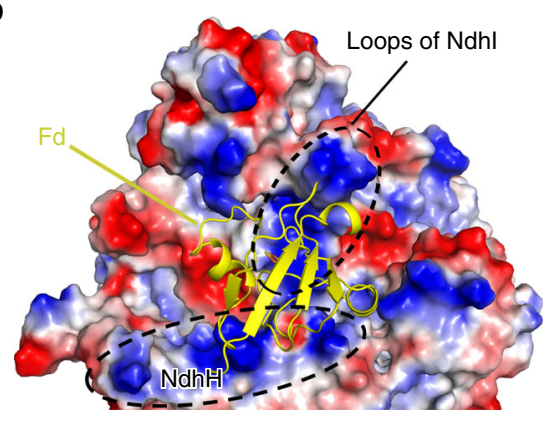

c

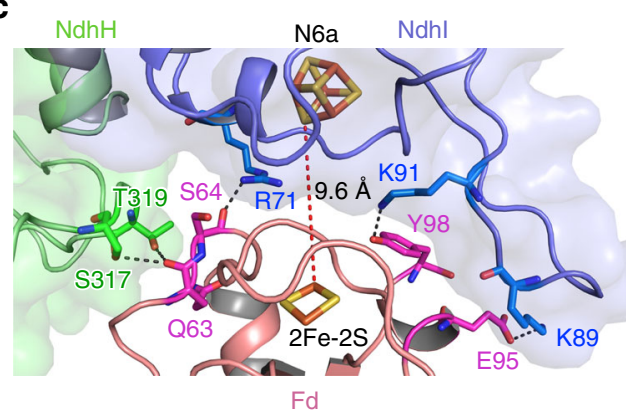

d

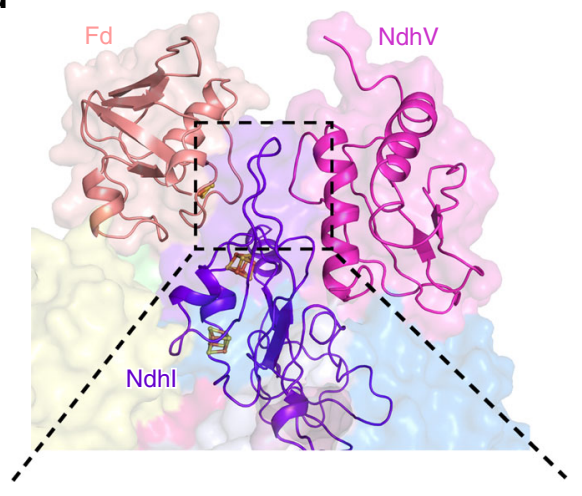

e

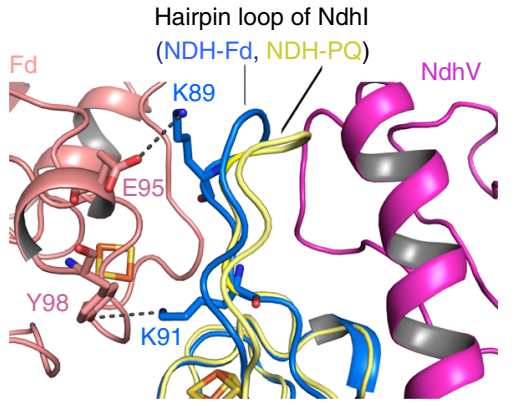

f

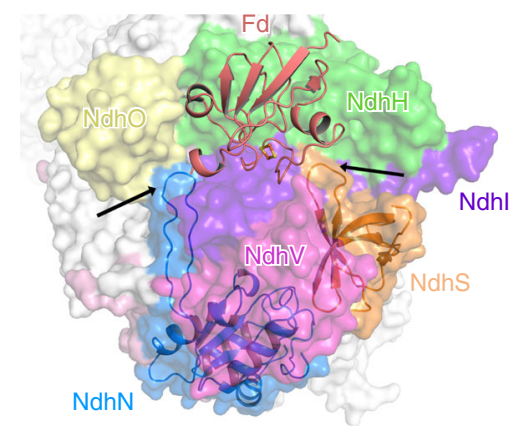

Fig. 4 The Fd binding pocket. a The Fd binding pocket is mainly formed by Ndhl and NdhH. Fd is shown in cartoon, the NDH subunits are shown in surface mode. Subunits close to Fd are colored differently and other subunits are shown in white. $\mathbf{b}$ The electrostatic surface representation of Fd binding pocket. Red represents negative charge, and blue represents positive charge. Fd is shown as yellow cartoon. The positively-charged regions surrounding Fd are highlighted with black dashed circle and labeled. c The interactions between Fd and Ndhl/NdhH subunits. The Fe-S clusters and the residues involved in the interactions between $\mathrm{Fd}$ and $\mathrm{Ndhl} / \mathrm{NdhH}$ are depicted as sticks and labeled. The hydrogen bonds are shown as black dashed lines. The distance between Fe-S clusters of Ndhl and Fd (red dashed line) is indicated. d Fd and NdhV almost symmetrically bind the hairpin loop of Ndhl from the opposite sides. The zoom-in view of the region circled by the black dashed line is shown in (e). e Superposition of NDH-Fd and NDH-PQ structures showing the different conformation of the hairpin loop in Ndhl subunit. Two positively-charged Lys residues from the hairpin loop of Ndhl are hydrogen bonded with residues from Fd. Residues involved in the interactions are shown as sticks and labeled. $\mathbf{f}$ The loop regions in NdhN and NdhS (pointed with black arrows) interact with $\mathrm{Ndhl}$ and/or $\mathrm{NdhH}$ and help to stabilize the Fd binding pocket.

from the N2 cluster (Fig. 5a). The PQ interacts with NdhA-A237 through one of its ketone group (Fig. 5c). In addition, E238 of NdhA is hydrogen bonded with residues from $\beta 1-2$ loop of $\mathrm{NdhH}$, including $\mathrm{H} 23$, which is further hydrogen bonded with NdhH-D124 (Fig. 5c). The PQ chamber is located at an area similar to that of the $\mathrm{Q}$ chamber identified from $\mathrm{Tt}$ complex I structure $^{38}$, but the $\mathrm{PQ}$ chamber in our structure is smaller than the previously assigned $\mathrm{Q}$ chamber at the upper part close to the $\mathrm{N} 2$ cluster (Fig. $5 \mathrm{~d}, \mathrm{e}$ ). The smaller PQ chamber is caused by the different conformation adopted by the $\beta 1-2$ loop of $\mathrm{NdhH}$, which intrudes into the PQ chamber, and the side chain of M22 blocks the path between PQ and the N2 cluster (Fig. 5d, f). In comparison, the corresponding $\beta 1-2$ loop in Tt complex I structure is away from the $\mathrm{Q}$ chamber, resulting in a larger chamber that is accessible to the N2 cluster (Fig. 5e, f). In agreement with that, the substrate/inhibitor in Q chamber in Tt complex I is located only $\sim 12 \AA$ from the $\mathrm{N} 2$ cluster, and interacts with residues Y87 and $\mathrm{H} 38$ of Nqo4 subunit (corresponding to NdhH-Y72 and NdhH$\mathrm{H} 23)^{38}$. These two residues were previously suggested to protonate quinone during the catalytic cycle. In addition, residue D139 of the Nqo4 subunit (corresponding to NdhH-D124) forms a hydrogen bond with $\mathrm{H} 38$, facilitating the protonation process ${ }^{38}$. These residues are completely conserved in the cyanobacterial $\mathrm{NDH}-1 \mathrm{~L}$, implying that similar interactions can also be formed in $\mathrm{NDH}-1 \mathrm{~L}$ complex. Indeed, the H23-D124 hydrogen bond is preserved in our NDH-PQ structure (Fig. 5c); however, the PQ molecule is more distantly located from the N2 cluster, without direct linking with $\mathrm{NdhH}-\mathrm{Y} 72$ and $\mathrm{NdhH}-\mathrm{H} 23$, implying that our structure represents a state of $\mathrm{NDH} /$ complex I that is not fully active. In addition to the $\beta 1-2$ loop of $\mathrm{NdhH}$, the TMH5-6 loop 
a

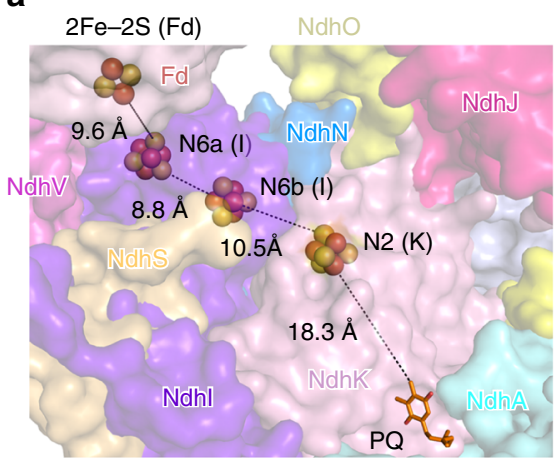

d

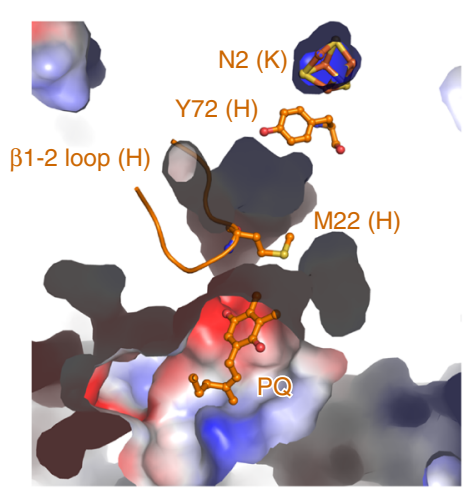

b

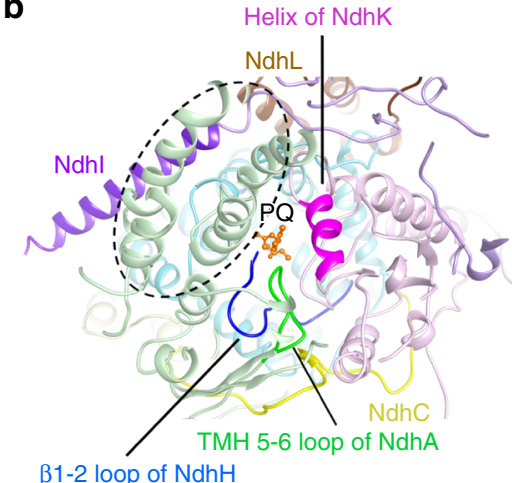

$\mathbf{e}$

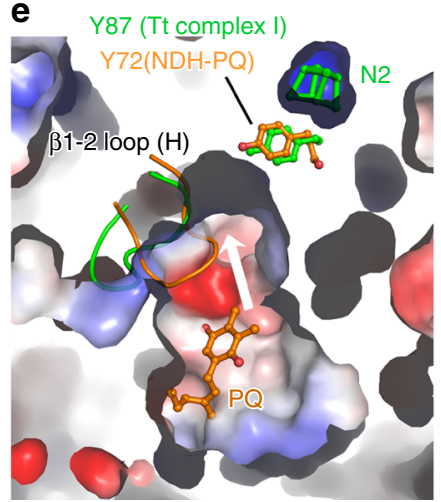

C

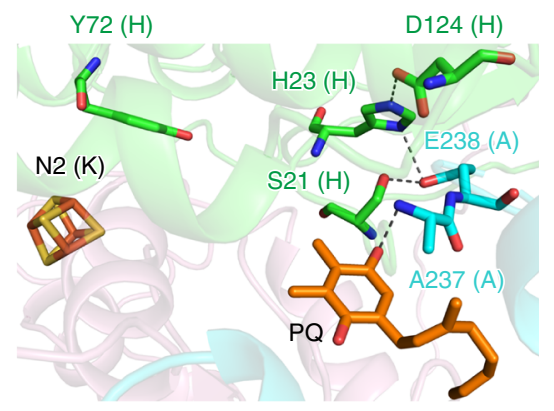

f

31-2 loop of $\mathrm{NdhH}$ (NDH-PQ, Tt complex I, YI complex I)

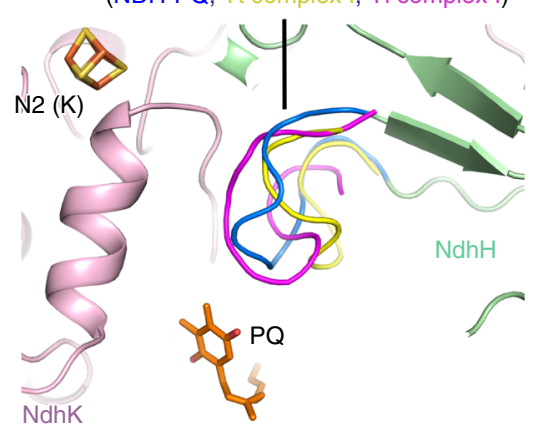

Fig. 5 The PQ chamber. a The cofactors involved in the electron transport chain. The PQ molecule is shown as sticks and the Fe-S clusters are shown as spheres, and the distances between cofactors are indicated. The subunits around PQ chamber are shown in surface with the same color code as that in Fig. 1. NdhH subunit is omitted for clarity. b The PQ chamber is shaped by NdhH, NdhK, and NdhA, which are further stabilized by Ndhl, NdhL, and NdhC. The structural elements involved in forming the PQ chamber are indicated. The four-helix bundle of NdhH is highlighted with black dashed circle. The PQ molecule is shown in ball-and-stick mode and colored orange. c The interactions around PQ molecules. The N2 cluster, PQ, and key residues are shown in sticks and labeled. Comparison of the PQ chamber in NDH-PQ structure (d) and the Q chamber in Tt complex I structure (PDB ID: 4HEA) (e) in the same view. In NDH-PQ structure, the $\beta 1-2$ loop containing $\mathrm{M} 22$ of $\mathrm{NdhH}$ intrudes into the PQ chamber and blocks the path from $\mathrm{PQ}$ to $\mathrm{Y} 72$ of $\mathrm{NdhH}$ and $\mathrm{N} 2$ cluster of NdhK, resulting in a smaller occluded PQ chamber (d), while the corresponding loop in Tt complex I structure (colored green) adopts a different conformation and results in a larger opened Q chamber (e). The NDH-PQ structure (colored orange) is superposed to Tt complex I structure (colored green) in $\mathbf{e}$, to show the positions of $\beta 1-2$ loop and $Y 72$ of $\mathrm{NdhH}$ as well as the PQ molecule in NDH-PQ structure. The white arrow indicates that a quinone molecule at the $\mathrm{PQ}$ position is able to move toward the N2 cluster in the larger PQ pocket as shown in the Tt complex I structure. $\mathbf{f}$ Comparison of the PQ/ Q chambers in NDH-PQ, Tt complex I (PDB ID: 4HEA), and YI complex I (PDB ID: 4WZ7) structures. The $\beta 1-2$ loop of NdhH in NDH-PQ structure and the corresponding loops in $\mathrm{Tt}$ and $\mathrm{YI}$ complex I structures are highlighted in different colors and labeled.

of NdhA in our NDH structure and the corresponding loops in complex I structures also exhibit variable conformations (Supplementary Fig. 8a). Moreover, the TMH5-6 loop of NdhA was not completely built in our NDH structure due to the very weak densities of a short fragment (residues L234-E236). Together, these results suggest that the two loops are highly flexible, and that they may adopt various conformations at different states and/or under different conditions.

Comparison of three NDH-1L structures. Together with the work presented here, three structures of NDH-1L with identical subunit composition are now available, with two from previous works (PDB codes $6 \mathrm{HUM}$ and $6 \mathrm{NBY})^{35,36}$. Interestingly, the three NDH complexes were purified under different $\mathrm{pH}$ conditions, thus these three structures are termed based on their purified $\mathrm{pH}$ values, namely $\mathrm{NDH}_{\mathrm{pH} 7}$ for our $\mathrm{NDH}-\mathrm{PQ}$ structure, and $\mathrm{NDH}_{\mathrm{pH} 8}(6 \mathrm{HUM})^{35}$ and $\mathrm{NDH}_{\mathrm{pH} 6}(6 \mathrm{NBY})^{36}$ for the previously reported structures. Structural comparison showed that the two loops around the PQ chamber, TMH5-6 loop of NdhA and $\beta 1-2$ loop of $\mathrm{NdhH}$, also adopt different conformations among the three NDH structures (Supplementary Fig. 8b). This result indicates the flexible nature of the loops constituting the PQ chamber ( $Q$ chamber), which might be associated with the physiological function of NDH-1L.

Structural comparison showed that $\mathrm{NDH}_{\mathrm{pH} 7}$ and $\mathrm{NDH}_{\mathrm{pH} 8}$ structures are relatively similar with each other, whereas $\mathrm{NDH}_{\mathrm{pH}} 6$ structure exhibits distinct local conformation of several subunits, which mainly occur at the interface between the membrane arm and the hydrophilic arm (Fig. 6a). The amphiphilic N-helix of NdhI stabilizes the membrane arm and simultaneously interacts with the soluble subunit $\mathrm{NdhH}$. The distance between $\mathrm{NdhH}$ and the N-helix of NdhI is the shortest in the $\mathrm{NDH}_{\mathrm{pH} 8}$ structure, but the longest in the $\mathrm{NDH}_{\mathrm{pH} 6}$ structure (Fig. 6b). Moreover, the TMH1-2 loop of NdhC, which is pivotal for connecting the membrane and the hydrophilic arms (Supplementary Fig. 6b) was well resolved, exhibiting similar conformations in $\mathrm{NDH}_{\mathrm{pH}}$ (Supplementary Fig. 4d) and $\mathrm{NDH}_{\mathrm{pH} 8}$ structures, but is invisible in $\mathrm{NDH}_{\mathrm{pH} 6}$ structure (Fig. 6c). In contrast to the TMH1-2 loop of $\mathrm{NdhC}$, the C-terminal fragment of $\mathrm{NdhG}$ in $\mathrm{NDH}_{\mathrm{pH} 6}$ structure is considerably stable and almost completely modeled. This fragment forms extensive hydrogen bonds with stromal residues of NdhB. In comparison, the C-terminal 30 residues of $\mathrm{NdhG}$ were not built in the other two structures (Fig. 6d). The structural 
a
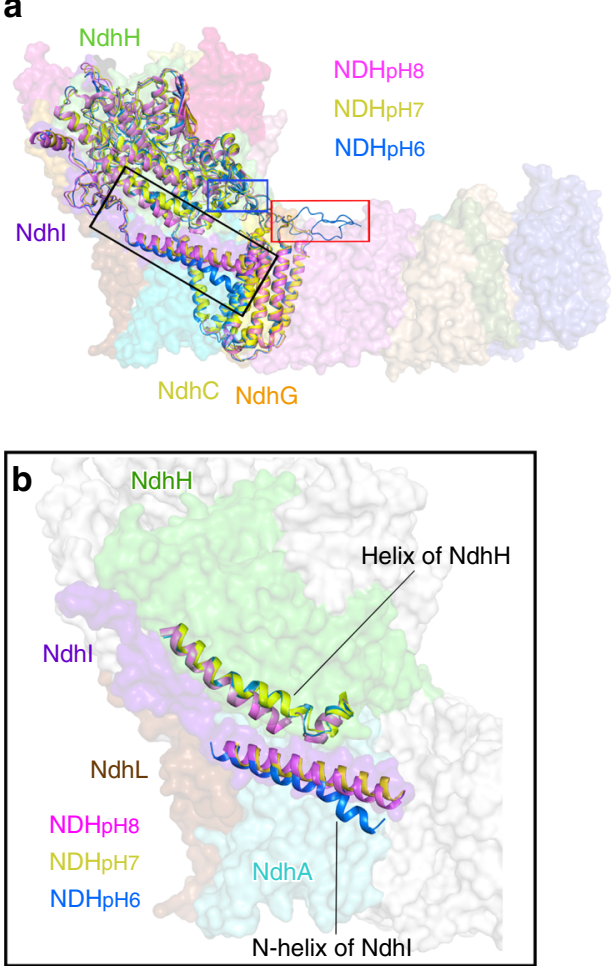
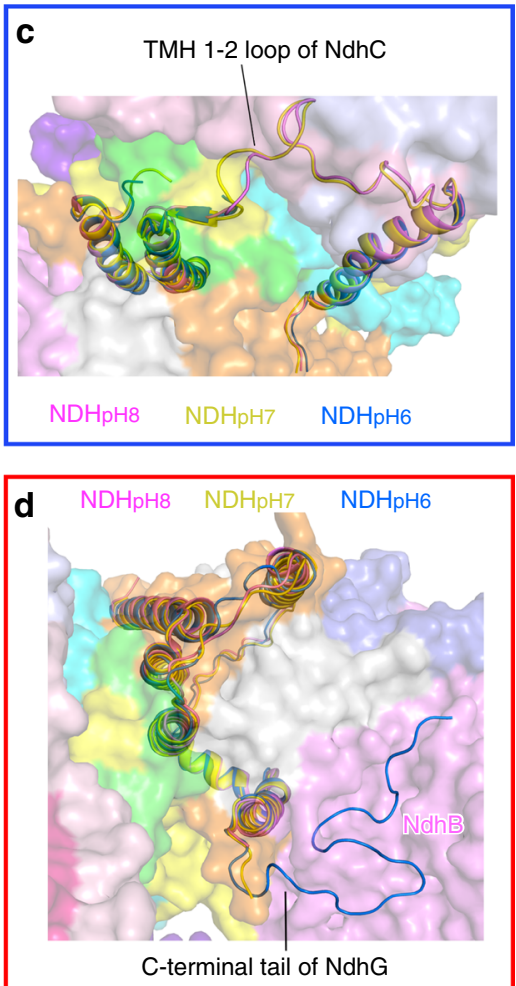

Fig. 6 Comparison of three $\mathbf{N D H - 1 L}$ structures under different pH conditions. a Comparison of NDH-PQ structure (NDH $\mathrm{pH}_{7}$ ) with previously reported two $\mathrm{NDH}-1 \mathrm{~L}$ structures $\mathrm{NDH}_{\mathrm{pH} 6}$ and $\mathrm{NDH}_{\mathrm{pH} 8}$ (PDB codes: $6 \mathrm{NBY}$ and $6 \mathrm{HUM}$ ) superposed on NdhA subunit. The subunits with structural differences are depicted as cartoons and labeled. The regions with evident differences among the three structures are circled with black, blue, and red boxes. b Comparison of the $\mathrm{Ndhl}$ and $\mathrm{NdhH}$ subunits. The $\mathrm{N}$-helix of $\mathrm{Ndhl}$ and a helix of $\mathrm{NdhH}$ are highlighted as cartoons. The subunits similar in three $\mathrm{NDH}-1 \mathrm{~L}$ structures are shown as surface mode (For clarity, only $\mathrm{NDH}_{\mathrm{pH}}$ structure is shown). comparison of $\mathrm{NdhC}$ subunits viewed from the stromal side. The TMH1-2 loop was not modeled in $\mathrm{NDH}_{\mathrm{pH} 6}$ structure. d Comparison of NdhG subunits viewed from the stromal side. Its $\mathrm{C}$-terminal tail in $\mathrm{NDH}_{\mathrm{pH} 6}$ structure is almost completely modeled and interacts with the stromal region of NdhB. Color codes are the same as that in Fig. 1.

comparison revealed that the $\mathrm{NDH}-1 \mathrm{~L}$ is more rigid in the membrane arm at low $\mathrm{pH}$, whereas the complex is more strongly associated between its two arms at higher $\mathrm{pH}$.

To evaluate the influence of $\mathrm{pH}$ on the function of $\mathrm{NDH}-1 \mathrm{~L}$, we further tested the CEF activity of the NDH-1L complex under various $\mathrm{pH}$ conditions (Supplementary Fig. 9). The CEF activity was monitored by measuring the postillumination increase in chlorophyll fluorescence, a method extensively used in previous reports $31,32,51-54$. Our results showed that NDH-1L exhibits the highest activity in mediating the $\mathrm{CEF}$ at $\mathrm{pH} 8.0$ and $\mathrm{pH} 7.0$, while at $\mathrm{pH} 6.0$, its activity drastically decreased (Supplementary Fig. 9c). These results implied the possibility that the close association between the membrane arm and the hydrophilic arm is related to the functionality of NDH-1L complex.

\section{Discussion}

The cryo-EM reconstructions of cyanobacterial NDH-1L complex presented here provide the complete structural model of NDH-1L and reveal the detailed binding pockets of Fd and PQ. Our structural analysis suggested that $\mathrm{NdhV}$ regulates the binding of Fd with NDH complex. In the NDH-Fd structure, NdhV bound to one side of the hairpin loop of NdhI may buttress the binding of Fd with NdhI at the opposite side (Fig. 4d, e). Thus, NdhV and Fd may bind NdhI in a synergistic manner. Previous report indicated that the level of $\mathrm{NdhV}$ is upregulated at high light ${ }^{31}$, which may increase the percentage of the NDH-1L complex containing NdhV, and further result in more Fd-bound NDH-1L. This can explain previous study showing that NDH-1 exhibits higher CEF activity at high light, which was suppressed by deletion of $\mathrm{NdhV}^{31}$. Therefore, the association of $\mathrm{NdhV}$ may finetune the activity of $\mathrm{NDH}$ complex by regulating its binding affinity with Fd.

The PQ chamber observed in our structure is located in an area close to the $\mathrm{Q}$ chamber in respiratory complex I. However, the B1-2 loop of $\mathrm{NdhH}$ intrudes into the PQ chamber, resulting in a smaller pocket that is occluded from the $\mathrm{N} 2$ cluster in our $\mathrm{NDH}$ PQ structure (Fig. 5d). The occluded feature of the PQ chamber, together with the long distance between PQ and the N2 cluster, implies that our structure represents an idle state of the $\mathrm{NDH}$ complex, as was previously suggested for the Yl complex I ${ }^{39}$. This complex also possesses a $\beta 1-2$ loop that intrudes into the $Q$ chamber (Fig. 5f). The idle state of the NDH complex is presumably pivotal for its functionality in the CEF process. During this process, ferredoxin shuttles only one electron to $\mathrm{NDH}$ complex (PQ) at a time, which probably results in the production of an unstable semiquinone radical intermediate $(\mathrm{PQ} \cdot \mathrm{H})$. The occluded PQ chamber may help to arrest the PQ molecule away from the N2 cluster, at least until the second electron of another Fd molecule is ready to be delivered. As a result, the PQ molecule can accept two electrons in a very short time range to produce $\mathrm{PQH}_{2}$. The close interaction of PQ with $\beta 1-2$ loop of $\mathrm{NdhH}$ (Fig. 5c) may coordinate the conformational change of the $\beta 1-2$ loop and a movement of PQ toward the N2 cluster. In addition, the $\beta 1-2$ loop of NdhH also interacts with the TMH5-6 loop of NdhA (Fig. 5c). The TMH5 of NdhA functions in proton translocation $^{38}$, and a number of conserved acidic residues (E226, E228, E236, E238, E239, and E240) are located in the C-terminal half of TMH5 and the TMH5-6 loop of NdhA. Therefore, changes induced by PQ movement could be further transferred to 
the proton pump modules, thus connecting PQ reduction and proton translocation.

\section{Methods}

Purification and characterization of NDH-1L. The cultivation of T. elongatus cells and purification of NDH-1L complex were performed according to the previous report $^{34}$ with some modifications. The wild type (T. elongatus BP-1) cells from visiting Prof. Ogawa were cultured in BG-11 media at $50^{\circ} \mathrm{C}$, bubbling with $\mathrm{CO}_{2}-$ enriched air (2\%) and illuminated with white light $\left(50-80 \mu \mathrm{mol}\right.$ photons $\left.\mathrm{m}^{-2} \mathrm{~s}^{-1}\right)$. Cells were harvested at logarithmic phase $\left(\mathrm{OD}_{730}=0.6-0.8\right)$ by centrifugation at $5000 \mathrm{~g}$ for $5 \mathrm{~min}$ at $4{ }^{\circ} \mathrm{C}$. The cell pellets were resuspended in buffer A $(20 \mathrm{mM}$ BisTris, pH 7.0, $10 \mathrm{mM} \mathrm{MgCl}, 10 \mathrm{mM} \mathrm{CaCl}_{2}, 20 \%$ glycerol), and then disrupted by French press (1300 bar, four times). The lysate was centrifuged at $5000 \mathrm{~g}$ for $10 \mathrm{~min}$ to remove unbroken cells. The supernatant was centrifuged at $150,000 \mathrm{~g}$ for $40 \mathrm{~min}$ (HITACHI, rotor P45AT) to harvest thylakoid membranes. The NDH-1L complex is able to be purified through $\mathrm{Ni}^{2+}$-affinity chromatography owing to the native histidine-rich area within the hydrophobic subunit NdhF1. Thus the thylakoid membranes were suspended in buffer B ( $20 \mathrm{mM}$ Bis-Tris, pH 7.0, $10 \mathrm{mM}$ $\mathrm{MgCl}_{2}, 20 \%$ glycerol) and were solubilized with $1 \%(\mathrm{w} / \mathrm{v}) \mathrm{n}$-dodecyl- $\beta$-D-maltoside $\left(\beta\right.$-DDM) at chlorophyll concentration of $1 \mathrm{mg} \mathrm{mL}^{-1}$. After gently stirring on ice for $1 \mathrm{~h}$, the solubilized membranes were centrifuged at $35,000 \mathrm{~g}$ for $30 \mathrm{~min}$ to remove the insoluble material. The supernatant was loaded onto a Ni-sepharose fast flow resin (GE Healthcare) pre-equilibrated with buffer C ( $20 \mathrm{mM}$ Bis-Tris, $\mathrm{pH}$ $7.0,100 \mathrm{mM} \mathrm{NaCl}, 0.03 \% \beta$-DDM). The resin was then washed with buffer $\mathrm{C}$ containing $20 \mathrm{mM}$ imidazole to remove the unbound proteins. The NDH-1L complex was subsequently eluted with buffer $\mathrm{C}$ containing $100 \mathrm{mM}$ imidazole. The eluted fraction was concentrated using a $100 \mathrm{kD}$-cut off spin concentrator. The concentrated protein was loaded onto a Superose 6 10/300 column (GE Healthcare) pre-equilibrated with buffer $\mathrm{C}$. The eluted fraction containing NDH-1L was analyzed by $10-15 \%$ gradient Tris-Tricine sodium dodecyl sulfate-polyacryamide gel electrophoresis in a vertical protein gel electrophoresis system (Bio-Rad) (Supplementary Fig. 1).

Expression and purification of two NDH-1L subunits and Fd. The NdhS and Fd gene of $T$. elongatus were amplified by PCR from T. elongatus CDNA using the

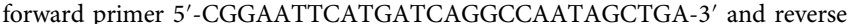
primer $5^{\prime}$-CCCAAGCTTTTACTTTTTCGCTTTCGCT-3' for NdhS, forward primer 5'-CGGAATTCATGGCAACCTACAAAGTAA-3' and reverse primer 5'-CC CAAGCTTTTAGTAAAGCTCTTCCTCTTGGT- $3^{\prime}$ for Fd. The NdhV gene was artificially synthesized (GenScript, China). The three genes were constructed to vector PET-28a with $\mathrm{N}$ - (NdhS and Fd) or C-(NdhV) His-tags, and were all transformed into E. coli strain BL21 (DE3) (Transgen, China). Cells were cultured in LB media with $50 \mu \mathrm{g} \mathrm{mL}^{-1}$ kanamycin and shaked at $37^{\circ} \mathrm{C}$. The protein expression was induced by adding $1 \mathrm{mM}$ IPTG (isopropyl $\beta$-D-1- thiogalactopyranoside) when $\mathrm{OD}_{600}$ reached 1.5 , while Fd required extra $1 \mathrm{mM} \mathrm{FeSO}_{4}$. The cells were further incubated at $16^{\circ} \mathrm{C}$ for $14 \mathrm{~h}$. These three proteins were purified with the same protocol as following. The cells were harvested by centrifugation at $8000 \mathrm{~g}$ for $6 \mathrm{~min}$. The cell pellets were resuspended in buffer D $(25 \mathrm{mM}$ Tris- $\mathrm{HCl}, \mathrm{pH} 8.0$, $300 \mathrm{mM} \mathrm{NaCl}, 20 \%$ glycerol, $1 \mathrm{mM}$ Tris(2-carboxyethyl)phosphine (TCEP)) and disrupted by ultrasonication. The lysate was centrifuged at $35,000 \mathrm{~g}$ for $30 \mathrm{~min}$, and the supernatant was loaded on to the Ni-sepharose fast flow resin (GE Healthcare), which was pre-equilibrated with buffer $\mathrm{D}$. After binding for about $1 \mathrm{~h}$, the resin was washed with 50 and $100 \mathrm{mM}$ imidazole in buffer $\mathrm{D}$, and the NdhS, NdhV, and Fd proteins were eluted with $250 \mathrm{mM}$ imidazole in buffer $\mathrm{D}$. The three proteins were further purified by size exclusion chromatography using a superdex $20010 / 300$ column (GE Healthcare) equilibrated with buffer E ( $25 \mathrm{mM}$ Tris-HCl pH 8.0, 300 $\mathrm{mM} \mathrm{NaCl}, 20 \%$ glycerol, $5 \mathrm{mM}$ Dithiothreitol (DTT)). The fractions containing NdhS, NdhV, and Fd were collected separately and concentrated to $5 \mathrm{mg} \mathrm{mL}^{-1}$ with using $3 \mathrm{kDa}$ cut off spin concentrators.

Preparation of NDH-Fd supercomplex. The purified NDH-1L complex was concentrated to $1 \mathrm{mg} \mathrm{mL}^{-1}$ and mixed with NdhS, NdhV, and Fd proteins. The molar ratio of NDH-1L:NdhS:NdhV:Fd was set to 1:4:6:6. The mixture was incubated for $1 \mathrm{~h}$ at $20^{\circ} \mathrm{C}$ and then concentrated to $12 \mathrm{mg} \mathrm{mL}^{-1}$ using a $100 \mathrm{kDa}$ cut off spin concentrator to remove the unbound small protein. The resulted NDH-Fd sample (NDH-1L in complex with Fd) was used for cryo-EM specimen preparation.

Grid preparation and data acquisition. Three microliter of NDH-1L (for solving the NDH-PQ structure) or NDH-Fd (for solving the NDH-Fd structure) samples at concentration of $12 \mathrm{mg} \mathrm{mL}^{-1}$ were applied to a glow-discharged holey carbon copper or gold grids (GIG, R1.2/1.3, 300 mesh). The grids were blotted for $2 \mathrm{~s}$ with blot force of level 2 at $95 \%$ humidity and $4{ }^{\circ} \mathrm{C}$, and were plunge frozen into liquid ethane cooled by liquid nitrogen immediately using a semiautomatic plunge device (Thermo Fisher Scientific Vitrobot IV). Sample screen was performed using Talos F200C $200 \mathrm{kV}$ or Talos L120C $120 \mathrm{kV}$ electron microscopes equipped with a Ceta camera (Thermo Fisher Scientific). For structure determination, the micrographs of both NDH-PQ and NDH-Fd were collected using SerialEM software suite ${ }^{55}$ on a $300 \mathrm{kV}$ Titan Krios electron microscope (Thermo Fisher Scientific) equipped with a
K2 camera (Gatan) and a GIF quantum energy filter (20 eV). Total of 1463 images for NDH-PQ and 2090 micrographs for NDH-Fd were acquired using a defocus range between 0.5 and $2.5 \mu \mathrm{m}$ at 130,000 magnification. The micrographs were exposed for $10 \mathrm{~s}$ and dose fractionated into 32 frames, leading to a total dose of $60 \mathrm{e}^{-} \AA^{-2}$

Image processing. Beam-induced motion of each movie stack was corrected by software MotionCor2 ${ }^{56}$, and the parameter of the contrast transfer function on each micrograph was determined by the program CTFFIND $4^{57}$. The software Relion-3.0 was used for further data processing ${ }^{58}$. For the NDH-PQ sample, about 1000 particles were manually picked and processed with reference-free $2 \mathrm{D}$ classification. Five class-averaged images were selected as references for subsequent particle auto-picking. A total of 434,021 particles were picked from 1463 micrographs. After 2D classification, 346,668 particles in good classes were kept for further data processing. A reference map was generated by the 3D-initial-model program in Relion-3.0 and the particles were classified into four classes by $3 \mathrm{D}$ classification. Class 1 containing 139,095 particles, is a NDH-PQ monomer, while class 3 containing 48,693 particles, is a NDH-PQ dimer. After refinement, the structures of NDH-PQ in either monomer or dimer showed the same conformational and structural features. The particles from the two classes were merged together for 3D autorefinement with a soft mask to mask monomer and resulted in a $3.3 \AA$ density map. After CTF refinement and another round of 3D autorefinement, the final resolution of NDH-PQ density map was improved to $3.0 \AA$ estimated based on the gold-standard Fourier shell correlation with 0.143 criterion (Supplementary Fig. 2). The data of NDH-Fd sample were processed in the same way as mentioned above. A total of 276,859 particles were picked from 2090 micrographs. After 2D classification, 246,088 NDH-Fd monomer particles were kept for 3D classification with the NDH-PQ map as the initial model. 152,003 particles were selected for further $3 \mathrm{D}$ autorefinement and resulted in a $3.20 \AA$ density map after CTF and beam-tilt refinement and beam-tilt estimation. To further improve the density of $\mathrm{Fd}, \mathrm{NdhV}$, and NdhS, focused refinement was used with a soft edged mask around the peripheral region, which resulted in resolution of $3.15 \AA$ of the peripheral region (Supplementary Fig. 3). The local resolution of the final map was calculated using ResMap ${ }^{59}$.

Model building and refinement. For the model building of subunits conserved in respiratory complex I (NdhA-NdhK), the homologous models were generated on the basis of the crystal structure of T. thermophilus complex I (PDB ID: 4HEA) ${ }^{38}$ by using software Chainsaw in the CCP4 software suite ${ }^{60}$. These homologous models were manually fitted in to the $3.0 \AA$ cryo-EM map of NDH-PQ one by one using UCSF chimera ${ }^{61}$. The OPS subunits NdhL-NdhQ were built and adjusted manually by $\operatorname{Coot}^{62}$ on the basis of the protein sequence and the predicted secondary structures. In the cryo-EM map of NDH-PQ, the density of NdhS subunit is very weak and the density of NdhV is invisible, but they behave well in the cryoEM map of NDH-Fd complex. Therefore the de novo model building of NdhS and NdhV were performed on the basis of the $3.15 \AA$ cryo-EM map of NDH-Fd complex. Regarding Fd protein, the crystal structure of Fd from T. elongatus BP-1 (PDB ID: $5 \mathrm{AUI})^{63}$ was manually fitted in to $3.15 \AA$ cryo-EM map of NDH-Fd using UCSF chimera. The initial models of NDH-PQ and NDH-Fd were refined in PHENIX-v1.15 $5^{64}$ using phenix.real_space_refine with geometry and secondary structure restraints. During real-space refinement, the distances between the iron atoms in the iron/sulfur clusters and the sulfur atom in the cysteine surrounded iron/sulfur clusters were constrained according to the values obtained from the high-resolution crystal structures. Automatic real-space refinements followed by manual correction in Coot were carried out interactively. The geometries of the final structures were assessed using MolProbity ${ }^{65}$. High-resolution images for publication were prepared using UCSF chimera and PyMOL (Molecular Graphics System, LLC).

Measurement of NDH activity in vivo. The postillumination increase in chlorophyll fluorescence was used to ascribe NDH-1L activity. The chlorophyll fluorescence was detected by a pulse-amplitude-modulation (PAM) chlorophyll fluorometer (Walz, Effeltrich, Germany), with emitter-detector-cuvette assembly (ED-101US), and an $101 \mathrm{ED}$ unit as described by Schreiber et al. ${ }^{66}$. The parameter Fo is a minimal fluorescence, which reflects the yield of Chl fluorescence when the reaction center is fully opened, and can be used to reflect the redox state of the primary electron acceptor PQ $(\mathrm{QA})$. The transient increase of Fo after termination of the actinic light is used to represent a reduction of PQ by photoreductants accumulated in cytosol during illumination, which is used to indirectly reflects the $\mathrm{CEF}$ activity of NDH. Cells were harvested at logarithmic phase $\left(\mathrm{OD}_{730}=0.6-0.8\right)$, and suspended in different $\mathrm{pH}$ buffer, $\mathrm{pH}$ 6.0 (20 mM HEPES), pH $7.0(20 \mathrm{mM}$ HEPES), pH 8.0 (20 mM Tris), pH 9.0 (20 mM Glycine), pH 10.0 (20 mM Glycine) before detected with PAM, and incubated in dark for 5 min before measurement Each experiment was independently repeated for four times and standard deviations were calculated.

SPR-based biosensor analysis. The interaction of the NDH-1L with Fd was detected by SPR using a Biacore T100 biosensor (GE Healthcare). The experiment was performed as described by He et al. ${ }^{34}$ with some modifications. NDH-1L was 
diluted with $10 \mathrm{mM}$ Glycine, $\mathrm{pH} 4.5$ to the concentration of $50 \mu \mathrm{gL}^{-1}$, and then immobilized on the activated CM5 chip surface to about $5000 \mathrm{RU}$. The noncovalently protein was removed with $2 \mathrm{M} \mathrm{NaCl}$. To compare the different interactions between NDH-1L and $\mathrm{Fd}$ in various $\mathrm{pH}$ conditions, three running buffers (50 $\mathrm{mM} \mathrm{NaH}_{2} \mathrm{PO}_{4}, 0.03 \% \beta$-DDM) with different $\mathrm{pH}(\mathrm{pH} 6.0, \mathrm{pH} 7.0$, and $\mathrm{pH} 8.0$ ) were prepared. A series of concentration of $\mathrm{Fd}(0.625,1.25,2.5,5,10$, and $20 \mu \mathrm{M})$ diluted separately with three running buffers were injected using a flow rate of $30 \mu \mathrm{L} \mathrm{min}{ }^{-1}$ for $1 \mathrm{~min}$. After 1 min dissociation, the bound $\mathrm{Fd}$ was washed off withv $2 \mathrm{M} \mathrm{NaCl}$. The data were analyzed with Biacore T100 evaluation software (GE Healthcare) by fitting to a 1:1 binding model.

Reporting summary. Further information on research design is available in the Nature Research Reporting Summary linked to this article.

\section{Data availability}

Data supporting the findings of this paper are available from the corresponding authors upon reasonable request. A reporting summary for this article is available as a Supplementary Information file. The source data underlying Supplementary Figs 1e, f, 7 , and $9 \mathrm{c}$ are provided as a Source Data file. The cryo-EM density maps have been deposited in the Electron Microscopy Data Bank (EMDB) under accession codes EMDB-9989 (for NDH-Fd structure) and EMDB-9990 (for NDH-PQ structure), and model coordinates have been deposited in the Protein Data Bank under accession number 6KHI (for NDH-Fd structure) and 6KHJ (for NDH-PQ structure).

Received: 25 September 2019; Accepted: 9 January 2020;

Published online: 30 January 2020

\section{References}

1. Rochaix, J. D. Regulation of photosynthetic electron transport. Biochim. Biophys. Acta 1807, 375-383 (2011).

2. Yamori, W. \& Shikanai, T. Physiological functions of cyclic electron transport around photosystem I in sustaining photosynthesis and plant growth. Annu. Rev. Plant Biol. 67, 81-106 (2016).

3. Johnson, M. P. Photosynthesis. Essays Biochem. 60, 255-273 (2016).

4. Shikanai, T. Central role of cyclic electron transport around photosystem I in the regulation of photosynthesis. Curr. Opin. Biotechnol. 26, 25-30 (2014).

5. Shikanai, T. Regulatory network of proton motive force: contribution of cyclic electron transport around photosystem I. Photosynth. Res. 129, 253-260 (2016).

6. Heber, U. Irrungen, Wirrungen? The Mehler reaction in relation to cyclic electron transport in C3 plants. Photosynth. Res. 73, 223-231 (2002).

7. Avenson, T. J., Cruz, J. A., Kanazawa, A. \& Kramer, D. M. Regulating the proton budget of higher plant photosynthesis. Proc. Natl Acad. Sci. USA 102, 9709-9713 (2005).

8. Joliot, P. \& Joliot, A. Cyclic electron flow in C3 plants. Biochim. Biophys. Acta 1757, 362-368 (2006).

9. Joliot, P. \& Johnson, G. N. Regulation of cyclic and linear electron flow in higher plants. Proc. Natl Acad. Sci. USA 108, 13317-13322 (2011).

10. Schurmann, P., Buchanan, B. B. \& Arnon, D. I. Role of cyclic photophosphorylation in photosynthetic carbon dioxide assimilation by isolated chloroplasts. Biochim. Biophys. Acta 267, 111-124 (1972).

11. Mills, J. D., Slovacek, R. E. \& Hind, G. Cyclic electron transport in isolated intact chloroplasts. Further studies with antimycin. Biochim. Biophys. Acta 504, 298-309 (1978)

12. Xu, M., Shi, N., Li, Q. \& Mi, H. An active supercomplex of NADPH dehydrogenase mediated cyclic electron flow around photosystem I from the panicle chloroplast of Oryza sativa. Acta Biochim. Biophys. Sin. 46, 757-765 (2014).

13. Takabayashi, A., Kishine, M., Asada, K., Endo, T. \& Sato, F. Differential use of two cyclic electron flows around photosystem I for driving $\mathrm{CO} 2$-concentration mechanism in C4 photosynthesis. Proc. Natl Acad. Sci. USA 102, 16898-16903 (2005).

14. Ishikawa, N. et al. NDH-mediated cyclic electron flow around photosystem I is crucial for C4 photosynthesis. Plant Cell Physiol. 57, 2020-2028 (2016).

15. Battchikova, N., Eisenhut, M. \& Aro, E. M. Cyanobacterial NDH-1 complexes: novel insights and remaining puzzles. Biochim. Biophys. Acta 1807, 935-944 (2011).

16. Heber, U. \& Walker, D. Concerning a dual function of coupled cyclic electron transport in leaves. Plant Physiol. 100, 1621-1626 (1992).

17. Endo, T., Shikanai, T., Takabayashi, A., Asada, K. \& Sato, F. The role of chloroplastic $\mathrm{NAD}(\mathrm{P}) \mathrm{H}$ dehydrogenase in photoprotection. FEBS Lett. 457, 5-8 (1999).

18. Mi, H., Deng, Y., Tanaka, Y., Hibino, T. \& Takabe, T. Photo-induction of an NADPH dehydrogenase which functions as a mediator of electron transport to the intersystem chain in the cyanobacterium Synechocystis PCC6803. Photosynth. Res. 70, 167-173 (2001).

19. Munekage, Y. et al. Cyclic electron flow around photosystem I is essential for photosynthesis. Nature 429, 579-582 (2004).

20. Peltier, G., Aro, E. M. \& Shikanai, T. NDH-1 and NDH-2 plastoquinone reductases in oxygenic photosynthesis. Annu. Rev. Plant Biol. 67, 55-80 (2016).

21. Strand, D. D., Fisher, N. \& Kramer, D. M. The higher plant plastid NAD(P)H dehydrogenase-like complex (NDH) is a high efficiency proton pump that increases ATP production by cyclic electron flow. J. Biol. Chem. 292, 11850-11860 (2017).

22. Friedrich, T., Steinmuller, K. \& Weiss, H. The proton-pumping respiratory complex I of bacteria and mitochondria and its homologue in chloroplasts. FEBS Lett. 367, 107-111 (1995).

23. Battchikova, N. \& Aro, E. M. Cyanobacterial NDH-1 complexes: multiplicity in function and subunit composition. Physiol. Plant 131, 22-32 (2007).

24. Ogawa, T. \& Mi, H. Cyanobacterial NADPH dehydrogenase complexes. Photosynth. Res. 93, 69-77 (2007).

25. Ma, W. \& Ogawa, T. Oxygenic photosynthesis-specific subunits of cyanobacterial NADPH dehydrogenases. IUBMB Life 67, 3-8 (2015).

26. Ogawa, $\mathrm{T}$. Identification and characterization of the ict $\mathrm{A} / \mathrm{ndhL}$ gene product essential to inorganic carbon transport of synechocystis PCC6803. Plant Physiol. 99, 1604-1608 (1992).

27. Prommeenate, P., Lennon, A. M., Markert, C., Hippler, M. \& Nixon, P. J. Subunit composition of NDH-1 complexes of Synechocystis sp. PCC 6803: identification of two new ndh gene products with nuclear-encoded homologues in the chloroplast Ndh complex. J. Biol. Chem. 279, 28165-28173 (2004).

28. Battchikova, N., Zhang, P., Rudd, S., Ogawa, T. \& Aro, E. M. Identification of NdhL and Ssl1690 (NdhO) in NDH-1L and NDH-1M complexes of Synechocystis sp. PCC 6803. J. Biol. Chem. 280, 2587-2595 (2005).

29. Nowaczyk, M. M. et al. NdhP and NdhQ: two novel small subunits of the cyanobacterial NDH-1 complex. Biochemistry 50, 1121-1124 (2011).

30. Battchikova, N. et al. Identification of novel Ssl0352 protein (NdhS), essentia for efficient operation of cyclic electron transport around photosystem I, in NADPH:plastoquinone oxidoreductase (NDH-1) complexes of Synechocystis sp. PCC 6803. J. Biol. Chem. 286, 36992-37001 (2011).

31. Chen, X., He, Z., Xu, M., Peng, L. \& Mi, H. NdhV subunit regulates the activity of type-1 $\mathrm{NAD}(\mathrm{P}) \mathrm{H}$ dehydrogenase under high light conditions in cyanobacterium Synechocystis sp. PCC 6803. Sci. Rep. 6, 28361 (2016).

32. Gao, F. et al. NdhV is a subunit of NADPH dehydrogenase essential for cyclic electron transport in Synechocystis sp. strain PCC 6803. Plant Physiol. 170, 752-760 (2016).

33. Wirth, C., Brandt, U., Hunte, C. \& Zickermann, V. Structure and function of mitochondrial complex I. Biochim. Biophys. Acta 1857, 902-914 (2016).

34. He, Z. et al. NDH-1L interacts with ferredoxin via the subunit NdhS in Thermosynechococcus elongatus. Photosynth. Res. 126, 341-349 (2015).

35. Schuller, J. M. et al. Structural adaptations of photosynthetic complex I enable ferredoxin-dependent electron transfer. Science 363, 257-260 (2019).

36. Laughlin, T. G., Bayne, A. N., Trempe, J. F., Savage, D. F. \& Davies, K. M. Structure of the complex I-like molecule NDH of oxygenic photosynthesis. Nature 566, 411-414 (2019).

37. Yamamoto, H., Peng, L., Fukao, Y. \& Shikanai, T. An Src homology 3 domainlike fold protein forms a ferredoxin binding site for the chloroplast NADH dehydrogenase-like complex in Arabidopsis. Plant Cell 23, 1480-1493 (2011).

38. Baradaran, R., Berrisford, J. M., Minhas, G. S. \& Sazanov, L. A. Crystal structure of the entire respiratory complex I. Nature 494, 443-448 (2013).

39. Zickermann, V. et al. Structural biology. Mechanistic insight from the crystal structure of mitochondrial complex I. Science 347, 44-49 (2015).

40. Verkhovsky, M., Bloch, D. A. \& Verkhovskaya, M. Tightly-bound ubiquinone in the Escherichia coli respiratory complex I. Biochim. Biophys. Acta 1817, 1550-1556 (2012).

41. Hashimoto, H., Uragami, C. \& Cogdell, R. J. Carotenoids and photosynthesis. Subcell. Biochem. 79, 111-139 (2016).

42. Herranen, M. et al. Towards functional proteomics of membrane protein complexes in Synechocystis sp. PCC 6803. Plant Physiol. 134, 470-481 (2004).

43. Zhang, P. et al. Isolation, subunit composition and interaction of the NDH-1 complexes from Thermosynechococcus elongatus BP-1. Biochem. J. 390, 513-520 (2005).

44. Zhang, J. et al. NdhP is an exclusive subunit of large complex of NADPH dehydrogenase essential to stabilize the complex in Synechocystis sp. strain PCC 6803. J. Biol. Chem. 289, 18770-18781 (2014).

45. Zhao, J., Rong, W., Gao, F., Ogawa, T. \& Ma, W. Subunit Q is required to stabilize the large complex of NADPH dehydrogenase in Synechocystis sp. strain PCC 6803. Plant Physiol. 168, 443-451 (2015).

46. Kurisu, G. et al. Structure of the electron transfer complex between ferredoxin and ferredoxin-NADP(+) reductase. Nat. Struct. Biol. 8, 117-121 (2001).

47. Dai, S. et al. Structural snapshots along the reaction pathway of ferredoxinthioredoxin reductase. Nature 448, 92-96 (2007). 
48. Srivastava, A. P. et al. Identification of the ferredoxin-binding site of a ferredoxin-dependent cyanobacterial nitrate reductase. Biochemistry 56, 5582-5592 (2017).

49. Page, C. C., Moser, C. C., Chen, X. \& Dutton, P. L. Natural engineering principles of electron tunnelling in biological oxidation-reduction. Nature 402, 47-52 (1999).

50. Mellor, S. B., Vavitsas, K., Nielsen, A. Z. \& Jensen, P. E. Photosynthetic fuel for heterologous enzymes: the role of electron carrier proteins. Photosynth. Res. 134, 329-342 (2017).

51. Mi, H. L., Endo, T., Ogawa, T. \& Asada, K. Thylakoid membrane-bound, nadph-specific pyridine-nucleotide dehydrogenase complex mediates cyclic electron-transport in the cyanobacterium Synechocystis Sp Pcc-68038. Plant Cell Physiol. 36, 661-668 (1995).

52. Deng, Y., Ye, J. \& Mi, H. Effects of low $\mathrm{CO} 2$ on NAD(P)H dehydrogenase, a mediator of cyclic electron transport around photosystem I in the cyanobacterium synechocystis PCC6803. Plant Cell Physiol. 44, 534-540 (2003).

53. Ma, W. M. \& Mi, H. L. Expression and activity of type 1 NAD(P)H dehydrogenase at different growth phases of the cyanobacterium, Synechocystis PCC 6803. Physiol Plant. 125, 135-140 (2005).

54. Kato, Y., Odahara, M., Fukao, Y. \& Shikanai, T. Stepwise evolution of supercomplex formation with photosystem I is required for stabilization of chloroplast NADH dehydrogenase-like complex: Lhca5-dependent supercomplex formation in Physcomitrella patens. Plant J. 96, 937-948 (2018).

55. Mastronarde, D. N. Automated electron microscope tomography using robust prediction of specimen movements. J. Struct. Biol. 152, 36-51 (2005).

56. Zheng, S. Q. et al. MotionCor2: anisotropic correction of beam-induced motion for improved cryo-electron microscopy. Nat. Methods 14, 331-332 (2017).

57. Rohou, A. \& Grigorieff, N. CTFFIND4: fast and accurate defocus estimation from electron micrographs. J. Struct. Biol. 192, 216-221 (2015).

58. Zivanov, J. \& Nakane, T. New tools for automated high-resolution cryo-EM structure determination in RELION-3. 7, e42166 (2018).

59. Kucukelbir, A., Sigworth, F. J. \& Tagare, H. D. Quantifying the local resolution of cryo-EM density maps. Nat. Methods 11, 63-65 (2014).

60. Winn, M. D. et al. Overview of the CCP4 suite and current developments. Acta Crystallogr. D. Biol. Crystallogr. 67, 235-242 (2011).

61. Pettersen, E. F. et al. UCSF Chimera-a visualization system for exploratory research and analysis. J. Comput. Chem. 25, 1605-1612 (2004).

62. Emsley, P., Lohkamp, B., Scott, W. G. \& Cowtan, K. Features and development of Coot. Acta Crystallogr. D. Biol. Crystallogr. 66, 486-501 (2010).

63. Mutoh, R. et al. X-ray structure and nuclear magnetic resonance analysis of the interaction sites of the Ga-substituted cyanobacterial ferredoxin. Biochemistry 54, 6052-6061 (2015).

64. Adams, P. D. et al. PHENIX: a comprehensive Python-based system for macromolecular structure solution. Acta Crystallogr. D. Biol. Crystallogr. 66, 213-221 (2010).

65. Chen, V. B. et al. MolProbity: all-atom structure validation for macromolecular crystallography. Acta Crystallogr. D. Biol. Crystallogr. 66 12-21 (2010).

66. Klughammer, C. \& Schreiber, U., Measuring, P700 absorbance changes in the near infrared spectral region with a dual wavelength pulse modulation system. Photosynthesis: Mechanisms and Effects, Vol. I-V, 4357-4360 (1998).

\section{Acknowledgements}

Cryo-EM data were collected at the Center for Biological Imaging, Core Facilities for Protein Science at the Institute of Biophysics (IBP), Chinese Academy of Sciences (CAS).
We thank B. Zhu, X. Huang, G. Ji, D. Fan, T. Niu, F. Sun, and other staff members at the Center for Biological Imaging (IBP, CAS) for the technical support in EM data collection; Y. Chen, Z. Yang, and B. Zhou for assisting in SPR experiments; L. Niu, Z. Xie, X. Ding, and M. Zhang and F. Yang for mass spectrometry; X. Zhao for assistance in culturing cyanobacteria; L. Shi for helping protein expression and purification; L. Kong for cryoEM data storage and backup. The authors thank Dr. Torsten Juelich from Peking University, China for linguistic assistance during the preparation of this manuscript. The project was funded by the National Key R\&D Program of China (2017YFA0503702, 2017YFA0504700, 2016YFA0502900), the Strategic Priority Research Program of CAS (XDB27020106, XDB08020302, XDB08030204), the Key Research Program of Frontier Sciences of CAS (QYZDB-SSW-SMC005), and National Natural Science Foundation of China (31930064, 31970264, 31770778, 31600609, 31770257). X.Z received scholarships from the "National Thousand (Young) Talents Program" from the Office of Global Experts Recruitment in China. X.P, D.C, and X.S are sponsored by the Youth Innovation Promotion Association at the Chinese Academy of Sciences.

\section{Author contributions}

M.L., H.M., X.Z., and W.C. conceived the project; F.Xie performed the sample preparation, characterization, and the SPR experiments; F.Xu performed the activity assay; D.C., X.P., X.S., and F.Xie collected the cryo-EM data; D.C. and X.Z. processed the cryoEM data and reconstructed the cryo-EM maps; X.P. built and refined the structure model; M.L., X.Z., and X.P. analyzed the structure; M.L., X.Z., and H.M. wrote the paper; all authors discussed and commented on the results and the paper.

\section{Competing interests}

The authors declare no competing interests.

\section{Additional information}

Supplementary information is available for this paper at https://doi.org/10.1038/s41467 020-14456-0.

Correspondence and requests for materials should be addressed to H.M., X.Z. or M.L.

Peer review information Nature Communications thanks Yuval Mazor and the other, anonymous, reviewer(s) for their contribution to the peer review of this work.

Reprints and permission information is available at http://www.nature.com/reprints

Publisher's note Springer Nature remains neutral with regard to jurisdictional claims in published maps and institutional affiliations.

\begin{abstract}
(c) (i) Open Access This article is licensed under a Creative Commons Attribution 4.0 International License, which permits use, sharing, adaptation, distribution and reproduction in any medium or format, as long as you give appropriate credit to the original author(s) and the source, provide a link to the Creative Commons license, and indicate if changes were made. The images or other third party material in this article are included in the article's Creative Commons license, unless indicated otherwise in a credit line to the material. If material is not included in the article's Creative Commons license and your intended use is not permitted by statutory regulation or exceeds the permitted use, you will need to obtain permission directly from the copyright holder. To view a copy of this license, visit http://creativecommons.org/ licenses/by/4.0/.
\end{abstract}

(C) The Author(s) 2020 